\title{
Medición de los resultados desde la perspectiva del paciente en cirugía de contorno corporal: creación del instrumento Body-QoL*
}

\author{
Drs. STEFAN DANILLA E. ${ }^{1}$, PEDRO CUEVAS T. ${ }^{1}$, MARÍA ELSA CALDERÓN G. ${ }^{1}$, \\ MARCO ANTONIO RÍOS V. ${ }^{1}$, CARLOS DOMÍNGUEZ C. ${ }^{1}$, \\ Soc. CRISTINA DI SILVESTRE P. ${ }^{2}$, Ints. JOSÉ IGNACIO VERGARA O. ${ }^{3}$, FELIPE SOTO V. ${ }^{3}$, \\ Als. PAULA SILVA R. ${ }^{4}$, SOFÍA SERRA D. ${ }^{4}$, SARAH AL-HIMDANI M.D. ${ }^{5}$, Dr. SERGIO SEPÚLVEDA P. ${ }^{1}$
}

1 Unidad de Cirugía Plástica, Departamento de Cirugía, Hospital Clínico de la Universidad de Chile.

2 Socióloga Facultad de Medicina, Dirección de Postgrado, Universidad del Desarrollo, Clínica Alemana.

3 Interno Facultad de Medicina Universidad de Chile.

4 Alumno Facultad de Medicina Universidad de Chile.

Santiago, Chile.

5 University Hospital of South Manchester, Manchester, UK.

\begin{abstract}
Development of an instrument to evaluate the results of plastic surgery from the perspective of the patient
\end{abstract}

Background: Body remodeling surgical procedures requires an assessment from the client's point of view in terms of esthetic satisfaction. Aim: To develop an instrument of assess body remodeling surgical results from the point of view of the patient. Material and Methods: A literature search for assessment methods was carried out. Posteriorly, in depth interviews and focus groups with plastic surgeons and patients subjected to plastic surgery procedures were performed. With this information, a conceptual framework was established to devise the instrument. Results: Sixteen patients and five plastic surgeons participated in the interviews and focus groups. The domains included in the conceptual framework were outfit and body image, affective and sexual life, self-image and esteem, social relationships and physical symptoms. Conclusions: The conceptual framework for the instrument is ready and the psychometric evaluation is the next step in its development.

Key words: Plastic surgery, body remodeling, patient assessment.

\section{Resumen}

Objetivo: Desarrollar un nuevo instrumento de resultados desde la perspectiva del paciente, diseñado para medir la satisfacción de éstos en relación a procedimientos de cirugía de contorno corporal, como lipoescultura, abdominoplastía, lifting corporal, lifting de muslos y brazos. Material y Método: Revisión de

*Recibido el 5 de mayo y aceptado el 6 de julio de 2013.

Los autores no refieren conflictos de interés.

Correspondencia: Stefan Danilla E.

Santos Dumont 999, Santiago, Chile. CP 8380456

drstefandanilla@gmail.com 
la literatura, entrevistas en profundidad a pacientes, grupos focales de discusión de expertos a cirujanos plásticos y pacientes sometidos a cirugías de contorno corporal con lo que se desarrolló un marco conceptual en relación a los resultados considerados importantes para la imagen corporal. Resultados: Dieciséis pacientes y cinco cirujanos plásticos fueron entrevistados. Los dominios identificados para el marco conceptual incluyeron vestimenta e imagen corporal, vida afectiva y sexual, autoimagen y autoestima, relaciones sociales y síntomas físicos. Conclusiones: Una vez completada la evaluación psicométrica, el instrumento Body-QoL y subescalas proporcionará una herramienta confiable para cirujanos plásticos, investigadores y pacientes para medir el impacto y la eficacia de los procedimientos de remodelamiento corporal, desde la perspectiva del paciente. Nivel de Evidencia: 1-Estudio cualitativo, generación de instrumentos de medición de resultado desde la perspectiva del paciente.

Palabras clave: Medicina basada en evidencia, instrumentos de medición, escalas de medición, cirugía de contorno corporal, abdominoplastía.

\section{Introducción}

Desde el inicio del desarrollo de la medicina basada en la evidencia (MBE), a mediados de la década de los ' $70 \mathrm{~s}^{1,2}$, la medicina clínica ha experimentado una explosión en la generación de conocimientos, mejorando así la calidad en distintas áreas de atención al paciente ${ }^{3}$. Actualmente los estudios de cohorte adecuadamente diseñados, así como los ensayos aleatorizados controlados constituyen el estándar para la adquisición del conocimiento de la historia natural de las enfermedades o la determinación de la eficacia y efectividad de nuevos tratamientos, como también de los ya existentes ${ }^{4}$.

En cirugía plástica estética de remodelamiento corporal, nuevas técnicas como la lipoabdominoplastía de Saldanha ${ }^{5}$, abdominoplastía de alta tensión lateral de Lockwood ${ }^{6}$, abdominoplastía con suturas de tensión progresiva de Baoroudi y Pollock ${ }^{7}$ Body Lift superior e inferior ${ }^{8,9}$, están cambiando los paradigmas clásicos de la cirugía. Además, nuevas tecnologías como lipolisis $\mathrm{LASER}^{8}$ y VASER ${ }^{9}$, radiofrecuencia ${ }^{10}$ y otros dispositivos, se presumen superiores en resultados estéticos, tiempo de recuperación y menos complicaciones al compararlos con técnicas clásicas, sin que hayan sido evaluados formalmente en ensayos clínicos controlados.

Generalmente, los resultados de cirugías cosméticas son presentados en publicaciones científicas como fotografías de antes y después; y hasta hace muy poco, no existían instrumentos desarrollados para medir los resultados desde la perspectiva del paciente.

Los resultados de investigación en cirugía plástica evalúan la perspectiva del paciente en relación a los resultados de la cirugía en una escala multidimensional, no sólo evaluando el resultado estético, sino también la mejora en el bienestar físico, psicológico y social después de la cirugía. La medición de resultados desde la perspectiva del paciente son cuestionarios altamente sofisticados que cuantifican la calidad de vida relacionada a la salud y otras variables importantes desde la perspectiva del paciente.

Pusic et al, desarrollaron el instrumento BREAST$Q^{11}$, diseñado para medir la calidad de vida relacionado con cirugías de reconstrucción, reducción y aumento mamario. Además, el mismo grupo, realizó el diseño preliminar del instrumento $F A C E-Q^{12}$, creado para medir resultados de blefaroplastía, lifting facial, rinoplastía, lifting cervical, lifting frontal e implante de mentón.

Nuestro grupo tradujo al español y validó el instrumento BREAST- $Q^{13}$, encontrando resultados altamente confiables en nuestra población.

El objetivo de nuestro grupo fue desarrollar un nuevo instrumento de resultados desde la perspectiva del paciente, llamado Body-QoL, separado en un conjunto de subescalas, abordando cada procedimiento de remodelamiento de contorno corporal y cada región anatómica como brazos, dorso, glúteos, cintura, muslos y abdomen (Figura 1).

Estas subescalas fueron diseñadas para medir una variedad de resultados que consideramos importantes en las cirugías de remodelamiento corporal, tanto para pacientes como a los distintos procedimientos quirúrgicos.

\section{Métodos}

Se siguieron guías internacionales de confección de instrumentos de evaluación de resultados desde la perspectiva del paciente para desarrollar el contenido de las escalas de cirugía de contorno corporal $^{14,15}$. En términos generales, un instrumento de evaluación de resultados desde la perspectiva del paciente, válido y confiable estadísticamente, debe ser desarrollado en tres fases. 1) marco conceptual y desarrollo preliminar de las escalas; 2) reducción de ítems y evaluación psicométrica y 3 ) prueba final del instrumento (Tabla 1).

El presente reporte corresponde a la primera fase de nuestro estudio. Nuestra meta fue generar un marco conceptual para la generación de dominios e 


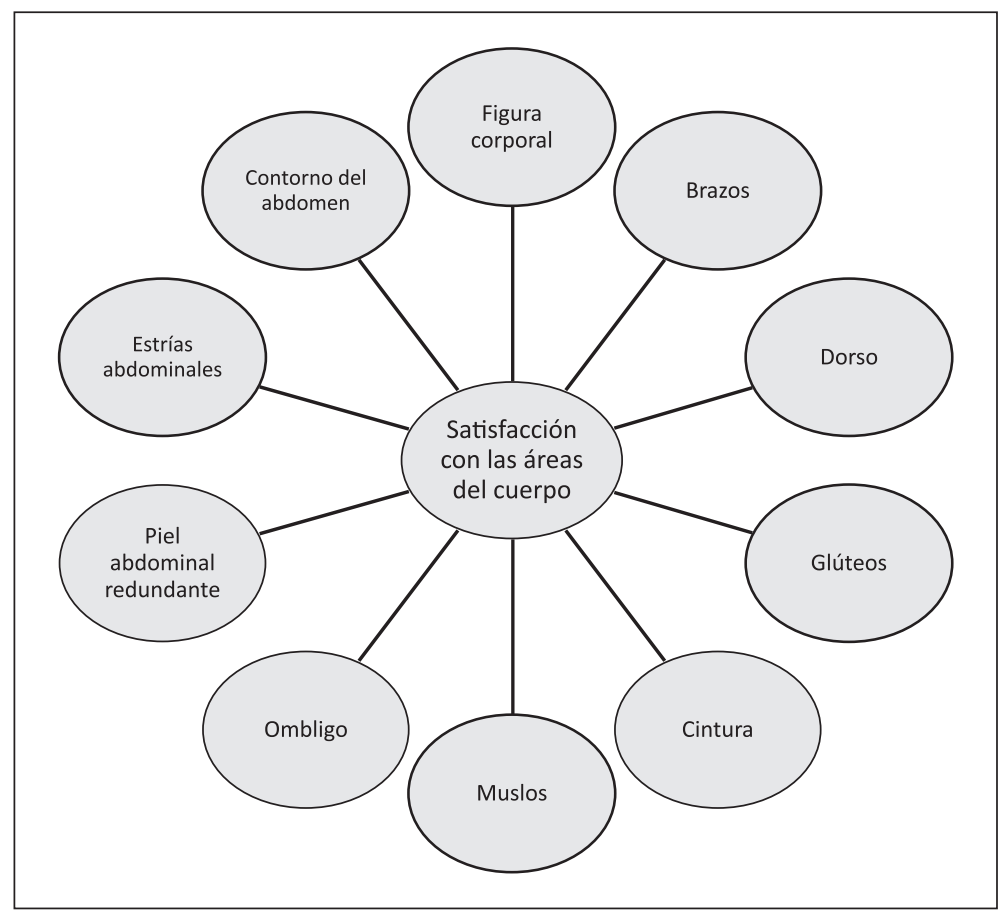

Figura 1. Marco conceptual sobre la satisfacción con las áreas del cuerpo.

Tabla 1. Fases de desarrollo de la medición de los resultados desde la perspectiva del paciente

Fase I Construcción de un marco conceptual, donde se incluyan todas las dimensiones del fenómeno a medir. Se desarrolla mediante una entrevista semi-estructurada realizada por un sociólogo entrenado, junto con la opinión de expertos y la revisión de la literatura. El instrumento preliminar se prueba en una muestra de pacientes para aclarar ambigüedades en la redacción de artículos, confirmar la pertinencia, y determinar la aceptabilidad y el tiempo total para completar el cuestionario

Fase II El cuestionario se aplica a una muestra amplia de pacientes para elegir los mejores ítems para su inclusión en el instrumento final. En esta fase, la evaluación psicométrica se lleva a cabo y la escala se reduce filtrando el cuestionario, para desarrollar un instrumento corto con los mejores ítems

Fase III Se lleva a cabo la evaluación psicométrica del instrumento final, incluyendo el coeficiente de confiabilidad, selección, validez, sensibilidad, especificidad, la correlación total ítems, etc. El objetivo es determinar las fortalezas y limitaciones del instrumento desarrollado

ítems, realizando una revisión exhaustiva de la literatura, discusión de grupos de expertos y entrevistas en profundidad a pacientes.

Utilizando un nuevo diseño cualitativo, realizamos entrevistas en profundidad con informantes clave. Los informantes clave fueron pacientes programados para cirugía de contorno corporal, incluyendo abdominoplastía, liposucción, lipo-abdominoplastía, lifting de muslos, brazos, lipectomía en cinturón y lifting corporal inferior.

Se realizaron entrevistas semi estructuradas por una socióloga entrenada (Master of Science en Epidemiología Clínica con vasta experiencia en investigación cualitativa); durante la entrevista, se permitió a los pacientes hablar libremente sobre sus motivaciones para realizar la cirugía, y además fueron interrogados específicamente sobre conceptos de belleza en relación a la forma corporal, sexualidad, autoimagen, autoestima, relaciones sociales y relaciones de trabajo (Tabla 2). Cada entrevista duró aproximadamente una hora; posteriormente, los pacientes fueron entrevistados tres meses luego de la cirugía, confrontando las creencias previas con la situación actual tras la cirugía.

El muestreo se llevó a cabo hasta la saturación de la información, de acuerdo a los estándares cualitativos de investigación: cuando los nuevos pacientes entrevistados no aportaron mayor información de 
Tabla 2. Guía para la entrevista en profundidad semi-estructurada

Razones para la cirugía: Influencia/opinión/percepción de la pareja, los amigos, familia y/o de la sociedad, la motivación, tipo de procedimiento elegido

Conceptos de belleza: Aspecto del cuerpo en general, detalles de la zona abdominal, el brazo y el muslo que se modificó, las preocupaciones de envejecimiento y el sobrepeso

Relación entre la belleza y la sexualidad: Bienestar psicológico y sexual y el concepto de sí mismo, el estado de ánimo, la confidencia con su desnudez, influencia de la imagen corporal en la vida sexual

Auto imagen corporal: Imagen con la ropa, autoestima, armonía corporal, forma de su cuerpo

Desempeño social: Actividades normales y laborales, impacto laboral, la capacidad de participar en los deportes/gimnasia/ actividades, cambio en el nivel de comodidad, energía y vitalidad

Relaciones sociales: Trato de amigos, colegas, compañeros de trabajo y familiares, discriminación en el trabajo

contenido que la ya recolectada, finalizamos el muestreo.

Las entrevistas fueron transcritas; luego, las afirmaciones significantes obtenidas de las transcripciones se agruparon en un agregado de temas comunes. Se desarrollaron ítems a partir de las afirmaciones significantes. Los ítems generados fueron agrupados en dominios surgidos de los agregados de temas comunes, construyendo la escala preliminar.

\section{Resultados}

Se entrevistaron un total de 16 pacientes, las características basales de los pacientes se muestran en la Tabla 3. De las entrevistas, se identificaron 201 afirmaciones significantes y se agruparon en 5 dominios y 93 ítems, conformando el instrumento preliminar. Un ejemplo del proceso de generación de un ítem a partir del proceso de entrevista se grafica en la Figura 2. Un esquema de los dominios de calidad de vida e ítems para el Body-QoL se muestran en la Figura 3.
Tabla 3. Características básicas de los pacientes entrevistados

\begin{tabular}{|lc|}
\hline Características & $\begin{array}{c}\text { Pacientes } \\
\text { entrevistados } \\
(\mathbf{n}=\mathbf{1 6})\end{array}$ \\
\hline Edad, años & \\
Promedio \pm Desviación estándar & $40,3 \pm 9,0$ \\
Rango intercuartil & 32 to 47 \\
Rango & 28 to 58 \\
Género & \\
Femenino & 15 \\
Masculino & 1 \\
Tipo de cirugía & \\
Abdominoplastía o lipo-abdominoplastía & 7 \\
Lipoescultura corporal & 3 \\
Lipectomía en cinturón & 2 \\
Levantamiento de muslo & 2 \\
Levantamiento corporal & 1 \\
Levantamiento de brazo & 1 \\
\hline
\end{tabular}

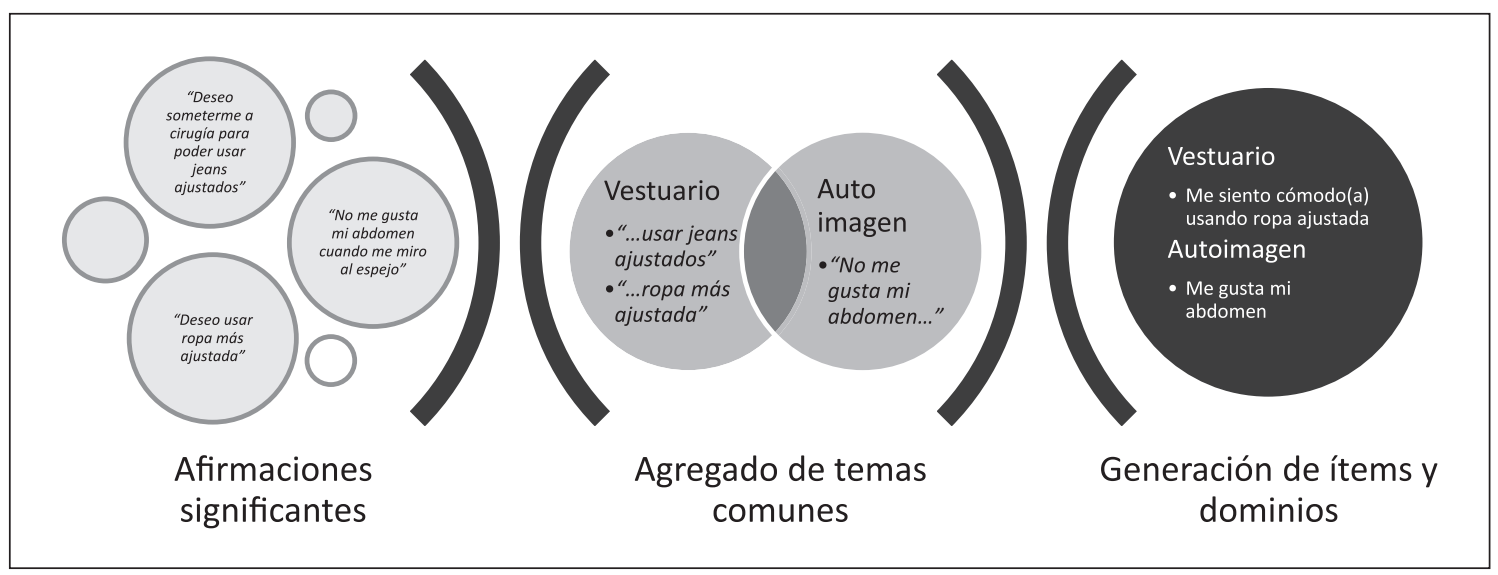

Figura 2. Representación esquemática de la generación de ítems y dominios. 
Los ítems fueron organizados como afirmaciones en escala de Likert de cinco puntos, en donde el paciente expresa su acuerdo con la afirmación, desde completamente de acuerdo hasta completamente en desacuerdo. En la Tabla 4 se muestra un ejemplo de dominios e ítems.
Los conceptos de "discapacidad postoperatoria" y la "evaluación técnica del cirujano" y "evaluación técnica del paciente" emergieron en el proceso de creación de la escala, por lo que dos nuevas subescalas fueron creadas; la primera, relacionada con dolor, incapacidad y recuperación luego de la ciru-

\begin{tabular}{|c|c|}
\hline $\begin{array}{c}\text { Vestuario e } \\
\text { imagen corporal }\end{array}$ & $\begin{array}{l}\text { - Comodidad usando ropa ajustada } \\
\text { - Comodidad ocupando ropa que deje ver partes del cuerpo } \\
\text { - Asuntos relacionados a la ropa interior }\end{array}$ \\
\hline $\begin{array}{l}\text { Vida sexual y } \\
\text { afectiva }\end{array}$ & $\begin{array}{l}\text { - Asuntos sexuales } \\
\text { - Relación con la pareja } \\
\text { - Confianza en la autoimagen estando desnudo(a) }\end{array}$ \\
\hline $\begin{array}{l}\text { Autoimagen y } \\
\text { autoestima }\end{array}$ & $\begin{array}{l}\text { - Me gusto } \\
\text { - Me siento atractivo(a) } \\
\text { - Estoy satisfecho(a) con mi cuerpo }\end{array}$ \\
\hline $\begin{array}{l}\text { Relaciones } \\
\text { sociales }\end{array}$ & $\begin{array}{l}\text { - Qué piensa la gente de mí } \\
\text { - Cómo me siento en sociedad } \\
\text { - La gente me encuentra atractivo(a) }\end{array}$ \\
\hline Síntomas físicos & $\begin{array}{l}\text { - Dolor lumbar } \\
\text { - Dermatitis en los pliegues abdominales } \\
\text { - Prúrito en el abdomen }\end{array}$ \\
\hline
\end{tabular}

Figura 3. Agregado de ítems y dominios.

Tabla 4. Ejemplo de los dominios e ítems del instrumento preliminar Body-QoL

\begin{tabular}{|c|c|c|c|c|}
\hline Dominio e ítem & $\begin{array}{c}\text { Completa- } \\
\text { mente de parte } \\
\text { acuerdo acuerdo }\end{array}$ & Indiferente & $\begin{array}{l}\text { En parte } \\
\text { en } \\
\text { desacuerdo }\end{array}$ & $\begin{array}{c}\text { Totalmente } \\
\text { en } \\
\text { desacuerdo }\end{array}$ \\
\hline $\begin{array}{l}\text { Vestuario y apariencia } \\
\text { Puedo usar la ropa que quiero } \\
\text { Trato de ocultar mi abdomen con mi ropa } \\
\text { Me siento cómodo(a) con ropa ajustada }\end{array}$ & & & & \\
\hline $\begin{array}{l}\text { Vida sexual y afectiva } \\
\text { Me gusta que mi pareja me vea desnudo(a) } \\
\text { Me siento atractivo(a) estando desnudo(a) } \\
\text { Siento vergüenza cuando mi pareja toca mi abdomen }\end{array}$ & & & & \\
\hline $\begin{array}{l}\text { Autoimagen y autoestima } \\
\text { Me siento bonito(a) } \\
\text { Me veo atractivo(a) vestido(a) } \\
\text { Mi abdomen cae }\end{array}$ & & & & \\
\hline $\begin{array}{l}\text { Entorno social } \\
\text { Me he sentido discriminado(a) en mi trabajo por mi apariencia } \\
\text { Mis conocidos me encuentran atractivo(a) } \\
\text { Me he sentido maltratado(a) a causa de mi cuerpo }\end{array}$ & & & & \\
\hline $\begin{array}{l}\text { Síntomas físicos } \\
\text { Mi ropa deja marcas en mi cuerpo } \\
\text { Siento dolor en la espalda baja } \\
\text { En verano presento hongos en los pliegues de mi guata }\end{array}$ & & & & \\
\hline
\end{tabular}




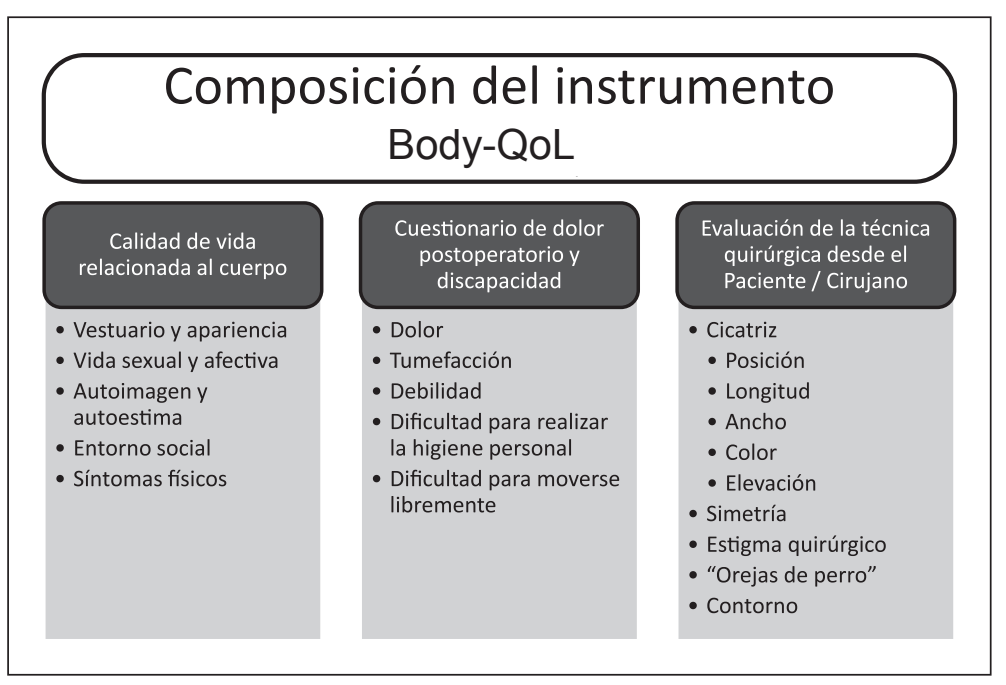

Figura 4. Composición del instrumento Body-QoL. gía; y la segunda alusiva a la evaluación del paciente y cirujano de los resultados técnicos de la cirugía, incluyendo longitud de cicatriz, ancho, simetría, naturalidad de los resultados, etc.

La versión preliminar del instrumento Body-QoL consta de tres módulos, los que se representan esquemáticamente en la Figura 4.

\section{Conclusiones}

La capacidad de medir la satisfacción del paciente con procedimientos quirúrgicos ha aumentado en importancia ${ }^{16,17}$. Desde un punto de vista académico, nos permite cuantificar objetivamente los resultados de una determinada técnica, así como también comparar entre técnicas ${ }^{18-20}$. Además, desde una perspectiva clínica, nos permite mejorar la relación cirujano-paciente, mostrándole al paciente su mejora, haciéndolos partícipes de su proceso de recuperación ${ }^{19,20}$.

En la primera fase de nuestro estudio, las entrevistas realizadas nos permitieron recolectar información valiosa, con lo que se pudo desarrollar la escala preliminar.

Los dominios desarrollados fueron consistentes y coherentes con el conocimiento previo y con publicaciones anteriores ${ }^{14,22}$.

El Body-QoL constituirá un instrumento robusto y completo, con subescalas para cada procedimiento de remodelación corporal. Además, tendrá 3 módulos: calidad de vida, escala de impacto de defectos en postoperatorio y evaluación paciente/cirujano de la técnica.

Una vez que las tres fases estén completas, la versión final del instrumento Body-QoL nos permitirá identificar "valores normales" para la población, medidas de la satisfacción del paciente con los procedimientos de remodelación corporal, el impacto de las tecnologías en los procedimientos en la calidad de vida y comparar entre distintas técnicas o dispositivos usados en los procedimientos de remodelación corporal.

Nuestro próximo reporte será luego de concluida la fase 2 de nuestro estudio, la que se encuentra en ejecución actualmente.

\section{Referencias}

1. Zimerman A. Evidence-based medicine: a short history of a modern medical movement. Virtual Mentor 2013;15:71-6.

2. Jeffrey A, Timothy C. History and Development of Evidence-based Medicine. World J of Surg. 2005;29:54753.

3. Manary M, Boulding W, Staelin R, Glickman S. The Patient Experience and Health Outcomes. Engl J Med. 2013;368:201-3.

4. Sackett D, Straus S, Richardson W. Rosenberg W, Haynes B. Evidence-Based Medicine: How to Practice and Teach EBM. 2nd ed. Edinburgh \& New York: Editorial Churchill Livingstone 2000;43-60.

5. Saldanha O, Pinto E, Matos W, Lucon R, Magalhães F, Bello E. Lipoabdominoplasty without undermining. Aesthet Surg J. 2001;21:518-26.

6. Lockwood T. Maximizing aesthetics in lateral-tension abdominoplasty and body lifts. Clin Plast Surg. 2004;31:523-37.

7. Andrades P, Prado A, Danilla S, Guerra C, Benítez S, Sepúlveda $\mathrm{S}$, et al. Progressive tension sutures in the prevention of postabdominoplasty seroma: a pros- 
pective, randomized, double-blind clinical trial. Plast Reconstr Surg. 2007;120:935-46.

8. Prado A, Andrades P, Danilla S, Leniz P, Castillo P, Gaete F. A prospective, randomized, double-blind, controlled clinical trial comparing laser-assisted lipoplasty with suction-assisted lipoplasty. Plast Reconstr Surg. 2006;118:1032-45.

9. Nagy M, Vanek P. A multicenter, prospective, randomized, single-blind, controlled clinical trial comparing VASER-assisted Lipoplasty and suction-assisted Lipoplasty. Plast Reconstr Surg. 2012;129:681-9.

10. Hurwitz D, Smith D. Treatment of overweight patients by radiofrequency-assisted liposuction (RFAL) for aesthetic reshaping and skin tightening. Aesthetic Plast Surg. 2012;36:62-71.

11. Pusic A, Klassen A, Scott A, Klok K, Cordeiro P, Cano P. Development of a new patient-reported outcome measure for breast surgery: the BREAST-Q. Plast Reconstr Surg. 2009;124:345-53.

12. Anne F, Klassen A, Phil D, Cano S, Scott A, Snell L, Pusic A. Measuring Patient-Reported Outcomes in Facial Aesthetic Patients: Development of the FACE-Q. Facial Plast Surg. 2010;26:303-9.

13. Cuevas P, Calderón M, Erazo C, Benítez S, Andrades $\mathrm{P}$, Sepúlveda S y cols. Mamoplastía de reducción: resultados desde la perspectiva del paciente. Validación lingüística y psicométrica del Breast Q Reduction and Mastopexy Module Instrument. Rev Chil Cir. 2013;65:146-9.

14. Scientific Advisory Committee of the Medical Outcomes Trust. Assessing health status and quality of life instruments: Attributes and review criteria. Qual Life Res. 2002;11:193-205.
15. U.S. Food and Drug Administration. Patient reported outcome measures: Use in medical product development to support labeling claims. FDA Guidance Compliance Regulatory Information. 2006. Disponible en: www. fda.gov/downloads/Drugs/GuidanceComplianceRegulatoryInformation/Guidances/ucm071975.pdf.

16. Kazis L, Anderson J, Meenan R. Effect sizes for interpreting changes in health status. Med Care 1989;27:17889.

17. Ramsey A, Larrabee W, Anderson S, Craig M, Calvin M. Measuring Cosmetic Facial Plastic Surgery Outcomes. Arch Facial Plast Surg. 2001;3:198-201.

18. Cano S, Klassen A, Pusic A. The science behind quality of- life measurement: A primer for plastic surgeons. Plast Reconstr Surg. 2009;123:98-106.

19. Thoma A, Veltri K, Khuthaila D, Rockwell G, Duku E. Comparison of the deep inferior epigastric perforator flap and free transverse rectus abdominis myocutaneous flap in postmastectomy reconstruction: A cost effectiveness analysis. Plast Reconstr Surg. 2004;113:1650-61.

20. Efficace F, Bottomley A, Osoba D, Gotay C, Flechtner $\mathrm{H}$, D'haese S, et al. Beyond the development of health related quality-of-life (HRQOL) measures: A checklist for evaluating HRQOL outcomes in cancer clinical trials. Does HRQOL evaluation in prostate cancer research inform clinical decision making? J Clin Oncol. 2003;21:3502-11.

21. Pusic A, Chen C, Cano S, Klassen A, McCarthy C, Collins D, Cordeir P. Measuring Quality of Life in Cosmetic and Reconstructive Breast Surgery: A Systematic Review of Patient-Reported Outcomes Instruments. Plastic and Reconstructive Surgery. 2007;120:823-37. 